\title{
A Keplerian disk around the Herbig Ae star HD 34282
}

\author{
V. Piétu, A. Dutrey, and C. Kahane \\ Laboratoire d'Astrophysique de Grenoble, BP 53, 38041 Grenoble Cedex 9, France \\ Received 9 July 2002 / Accepted 18 September 2002

\begin{abstract}
We report new millimeter observations of the circumstellar material surrounding the Herbig Ae A0.5 star HD 34282 performed with the IRAM array in $\mathrm{CO} J=2 \rightarrow 1$ and in continuum at $1.3 \mathrm{~mm}$. These observations have revealed the existence of a large Keplerian disk around the star. We have analysed simultaneously the line and continuum emissions to derive the physical properties of both the gas and the dust. The analysis of our observations also shows that the Hipparcos distance to the star is somewhat underestimated; the actual distance is probably about $400 \mathrm{pc}$. With this distance the disk around HD 34282 appears more massive and somewhat hotter than the observed disks around less massive $\mathrm{T}$ Tauri stars, but shares the general behaviour of passive disks.
\end{abstract}

Key words. stars: individual: HD 34282 - stars: planetary systems: protoplanetary disks - stars: pre-main sequence stars: distances - radio lines: stars - radio continuum: stars

\section{Introduction}

Interferometric CO line observations of $\mathrm{T}$ Tauri stars in the Taurus-Auriga cloud clearly demonstrate that many lowmass Pre-Main-Sequence (PMS) stars are surrounded by large $\left(R_{\text {out }} \sim\right.$ 200-800 AU) Keplerian disks (Koerner et al. 1993; Dutrey et al. 1994). There is little comparable evidence for such disks around intermediate-mass PMS objects, the Herbig Ae stars. One example of a molecular disk around an A-type star is MWC480 (spectral type A4) which possesses a large CO disk (Mannings et al. 1997). Near-infrared observations reveal that Herbig Ae stars can be surrounded by large reflection nebulae as in the case of AB Auriga (Grady et al. 1999), while ISO data provide very strong evidence for disk geometries (e.g. AB Aur and HD 163296, Bouwman et al. 2000). However, to date, there is little known about the molecular content and dynamics of these systems.

In this context, the A0 star HD $34282(\alpha=05: 16: 00.47$, $\delta=-09: 48: 35.3, \mathrm{~J} 2000.0)$ appears a very interesting object because i) the star has a strong IR excess (Sylvester et al. 1996; Malfait et al. 1998), ii) it is a nearby star, according to Hipparcos measurements $D=160_{-40}^{+60} \mathrm{pc}$, (van den Ancker et al. 1998) and iii) single-dish observations performed with the IRAM $30 \mathrm{~m}$ telescope (in November 1998) revealed a CO $J=2 \rightarrow 1$ double peak profile, strongly suggestive of a rotating disk, also observed by Greaves et al. (2000) in the CO $J=3 \rightarrow 2$ JCMT spectrum. Moreover, Malfait et al. (1998) reported optical variability of the order of $2.5 \mathrm{mag}$ in the $V$ band, which suggests that HD 34282 might be an UX Orionis-type star.

Send offprint requests to: V. Piétu, e-mail: Vincent.Pietu@obs.ujf-grenoble.fr
Interferometric observations of $\mathrm{CO}$ rotational lines remain the best tool to search for large cold Keplerian disks similar to those found around T Tauri stars, while millimeter continuum emission provides complementary information on the dust. We used the IRAM interferometer $(\mathrm{PdBI})^{1}$ to map the circumstellar material around HD 34282 simultaneously in ${ }^{12} \mathrm{CO} J=2 \rightarrow 1$ at $1.3 \mathrm{~mm}$ and in continuum at 3.4 and $1.3 \mathrm{~mm}$.

\section{Observational data}

The CO $J=2 \rightarrow 1$ and the continuum emissions from HD 34282 were mapped with the PdBI, using three configurations: 4D1, in August 1999, 4B1 and 4C2+N09 in October 1999. Due to the low elevation of the source, the resulting beam is strongly elliptical $\left(2.31^{\prime \prime} \times 1.42^{\prime \prime}\right.$ at $\left.\mathrm{PA}=20^{\circ}\right)$. The spectral resolution was $0.10 \mathrm{~km} \mathrm{~s}^{-1}$ per channel at the $\mathrm{CO}$ line frequency. We used full correlator power to look simultaneously for dust continuum emission at 1.3 and $3.4 \mathrm{~mm}$. Data were reduced using the GILDAS package at IRAM Grenoble. At $1.3 \mathrm{~mm}$, the seeing was about $0.3^{\prime \prime}$. Dirty maps were deconvolved using the classical CLEAN algorithm. The integrated flux derived from the $\mathrm{CO} J=2 \rightarrow 1$ spectrum observed at the IRAM 30 m telescope (Pico Veleta, Spain) in November 1998 is $4.8 \pm 0.4 \mathrm{Jy} \mathrm{km} \mathrm{s}^{-1}$, while the interferometric map leads to an integrated flux of $4.2 \pm 0.1 \mathrm{Jy} \mathrm{km} \mathrm{s}^{-1}$. As the two fluxes are in good agreement, we are confident in the interferometric flux calibration, and we conclude that our interferometric observations do not miss much flux.

\footnotetext{
${ }^{1}$ Based on observations carried out with the IRAM Plateau de Bure Interferometer. IRAM is supported by INSU/CNRS (France), MPG (Germany) and IGN (Spain).
} 


\section{Data analysis}

\subsection{Millimeter SED}

Following Beckwith et al. (1990), we write the dust absorption coefficient as $\kappa_{\mathrm{d}}(v)=0.1\left(v / 10^{12} \mathrm{~Hz}\right)^{\beta} \mathrm{cm}^{2} \mathrm{~g}^{-1}$. As the spectral index derived from the fluxes reported in Table 1 is ( $\alpha=3.15 \pm 0.20$ ), we conclude that thermal dust emission from the circumstellar material around HD 34282 is optically thin at millimeter wavelengths. The corresponding dust opacity spectral index, $\beta=1.15 \pm 0.20$, is significantly smaller than the spectral index of 2 (Beckwith et al. 1990) measured in the interstellar medium. The same behaviour is observed in T Tauri's disks (Dutrey et al. 1996). This similarly suggests that grain growth is occuring in the disk.

Table 1. Continuum fluxes of HD 34282.

\begin{tabular}{ccl}
\hline \hline $\begin{array}{c}\lambda \\
(\mathrm{mm})\end{array}$ & $\begin{array}{c}F_{\lambda} \\
(\mathrm{mJy})\end{array}$ & References \\
\hline 1.1 & $183 \pm 17$ & (Sylvester et al. 1996) \\
1.3 & $110 \pm 10$ & This work $^{a}$ \\
2.6 & $23.8 \pm 3.0$ & (Mannings \& Sargent 2000) \\
3.4 & $5.0 \pm 0.3$ & This work \\
\hline
\end{tabular}

$a$ We fitted an apparent size of $1.74 \pm 0.07^{\prime \prime} \times 0.89 \pm 0.06^{\prime \prime}$ at $1.3 \mathrm{~mm}$. The disk is unresolved at $3.4 \mathrm{~mm}$.

\subsection{Dust and CO PdBI data modeling}

We present in this section an improvement of the method described in Guilloteau \& Dutrey (1998). It is based on a $\chi^{2}$ minimization in the $u v$ plane of a standard disk model, as we describe below. In the improved method, we combine the ${ }^{12} \mathrm{CO} J=2 \rightarrow 1$ line and the $1.3 \mathrm{~mm}$ continuum analysis in an iterative way. Assuming the ${ }^{12} \mathrm{CO} J=2 \rightarrow 1$ line is optically thick, and the continuum at $1.3 \mathrm{~mm}$ is mainly optically thin (see previous section), the method described below enables us to derive both temperature and density distributions with some "classical" assumptions: the dust and the gas have the same temperature, the gas to dust ratio is 100 , the dust opacity at $10^{12} \mathrm{~Hz}$ is $0.1 \mathrm{~cm}^{2} \mathrm{~g}^{-1}$ and its spectral index $\beta$ is the one derived above.

The disk is assumed to be in local hydrostatic equilibrium, and the physical conditions in the disk are assumed to follow local thermodynamic equilibrium. The physical parameters are described as power laws with the following radial dependences: $n(r)=n_{100} \times(r / 100 \mathrm{AU})^{-s}$ for $\mathrm{H}_{2}$ density, $T(r)=T_{100} \times(r / 100 \mathrm{AU})^{-q}$ for kinetic temperature and $v(r)=v_{100} \times(r / 100 \mathrm{AU})^{-v}$ for rotation velocity. The surface density, $\Sigma(r)=\Sigma_{100} \times(r / 100 \mathrm{AU})^{-p}$, can be deduced from the scale height $H(r)=H_{100} \times(r / 100 \mathrm{AU})^{+h}$ and the density law $n(r)$ via $n(r)=\Sigma(r) / \sqrt{\pi} H(r)$.

For optically thick ${ }^{12} \mathrm{CO} J=2 \rightarrow 1$ emission, we cannot measure the density distribution, but we are able to constrain the disk geometry (the outer radius $R_{\text {out }}$ and inclination $i$ ), the temperature profile and the turbulent velocity $\Delta v$ in the disk. Assuming a distance $D$, we first perform $\chi^{2}$ minimizations in the 2-dimensional parameter spaces (PA, $V_{\mathrm{lsr}}$ ), $\left(V_{100}, v\right),\left(V_{100} \sin i, i\right),\left(T_{100}, q\right)$ and $\left(\Delta v, R_{\text {out }}\right)$ and then in the 5-dimensional and 4-dimensional spaces $\left(T_{100}, q, R_{\text {out }}, i\right.$ and $\left.V_{100} \sin i\right)$ and $\left(T_{100}, q, R_{\text {out }}, \Delta v\right)$, which correspond to the more coupled parameters. The most important results of the minimizations are presented in Fig. 1. They will be discussed in next section.
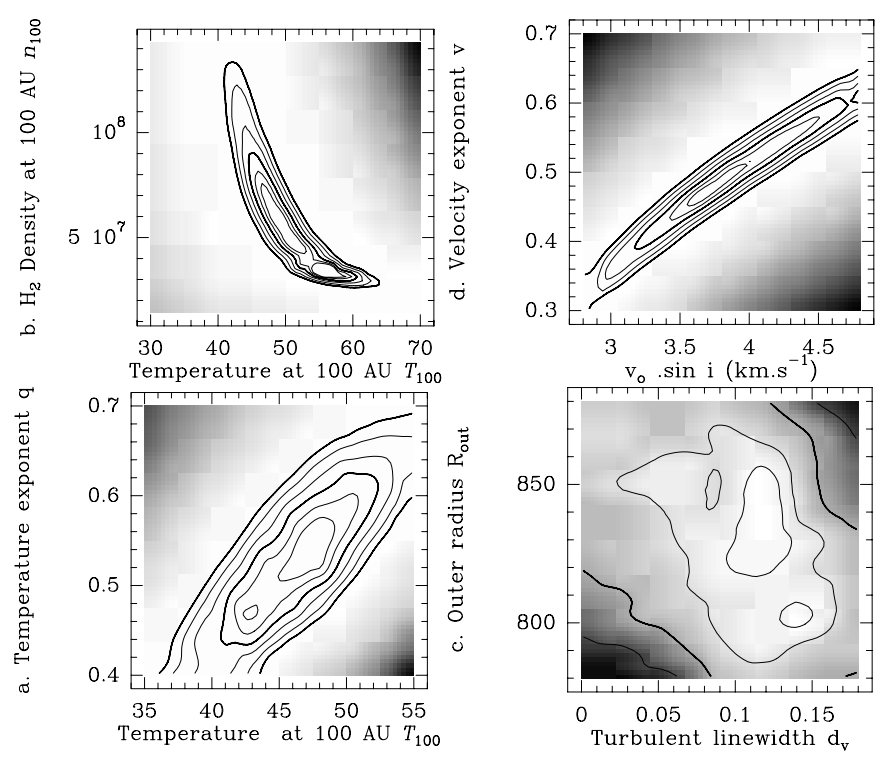

Fig. 1. Examples of the $\mathrm{CO}$ iso- $\chi^{2}$ surfaces. The contours run from 1 to $6 \sigma$. Top panels: $\left(T_{100}, n_{100}\right)$ and $\left(V_{100} \sin i, v\right)$ are coming from the $2 \times 2$ minimization. Bottom panels: $\left(T_{100}, q\right)$ and $\left(\Delta v, R_{\text {out }}\right)$ are from the final $4 \times 4$ minimization assuming the rotation pattern is Keplerian $(v=0.5)$ and the density $n_{100}$ (dust) (see text).

A $\chi^{2}$ minimization performed on the parameters $\left(n_{100}, T_{100}\right)$ shows that the line is optically thick; above a threshold called $n_{100}(\mathrm{CO})$ in the following, the temperature becomes almost independent of the density. According to Fig. $1 \mathrm{~b}, n_{100}(\mathrm{CO})$ is close to the mimimum of the $\chi^{2}$ map $\left(n_{100}(\mathrm{CO}), T_{100}(\mathrm{CO})\right)$ (note that this $\mathrm{H}_{2}$ density assumes a standard $\mathrm{CO}$ abundance $\left.X(\mathrm{CO})=X_{\mathrm{TMC} 1}^{12}=7 \times 10^{-5}\right)$.

As the disk dust thermal emission is at least marginally resolved, we may obtain an independent estimate of the surface density (and the density, since the geometry is constrained) from the optically thin continuum map at $1.3 \mathrm{~mm}$. Optically thin dust emissivity is $\propto T_{100} \times \Sigma_{100} \times r^{-(p+q)}$. Following the DM Tau disk analysis by Dartois et al. (in prep.) we have performed a minimization on the parameters $\left(p+q, \Sigma_{100} \times T_{100}, p-q\right.$, $\left.\Sigma_{100} / T_{100}\right)$. The results, given in Fig. 2 , confirm that dust emission is optically thin (since $p+q$ and $\Sigma_{100} \times T_{100}$ are relatively well constrained). Assuming the dust temperature law derived from ${ }^{12} \mathrm{CO}$, we thus can derive $p$ and $\Sigma_{100}$ (or $s$ and $n_{100}$ ).

Comparison of the $\mathrm{H}_{2}$ density lower limit, derived from the ${ }^{12} \mathrm{CO}$ with the $\mathrm{H}_{2}$ density derived from dust emission $n_{100}$ (dust), provides an lower limit to the $\mathrm{CO}$ abundance: $X(\mathrm{CO}) \geq n_{100}(\mathrm{CO}) / n_{100}$ (dust) $\times X_{\mathrm{TMC} 1}^{12} \sim 10^{-6}$. Equivalently, it also corresponds to an upper limit to the 

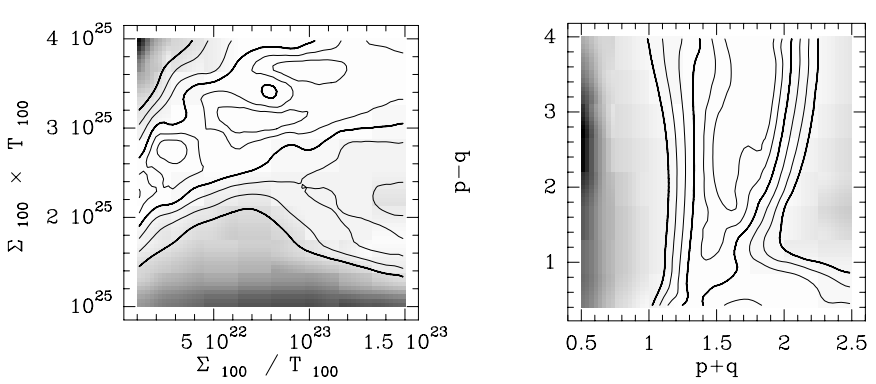

Fig. 2. Dust iso- $\chi^{2}$ surface. Contours run from 1 to $6 \sigma$. Left: $\left(\Sigma_{100} \times\right.$ $\left.T_{100}, \Sigma_{100} / T_{100}\right)$; right: $(p+q, p-q)$.

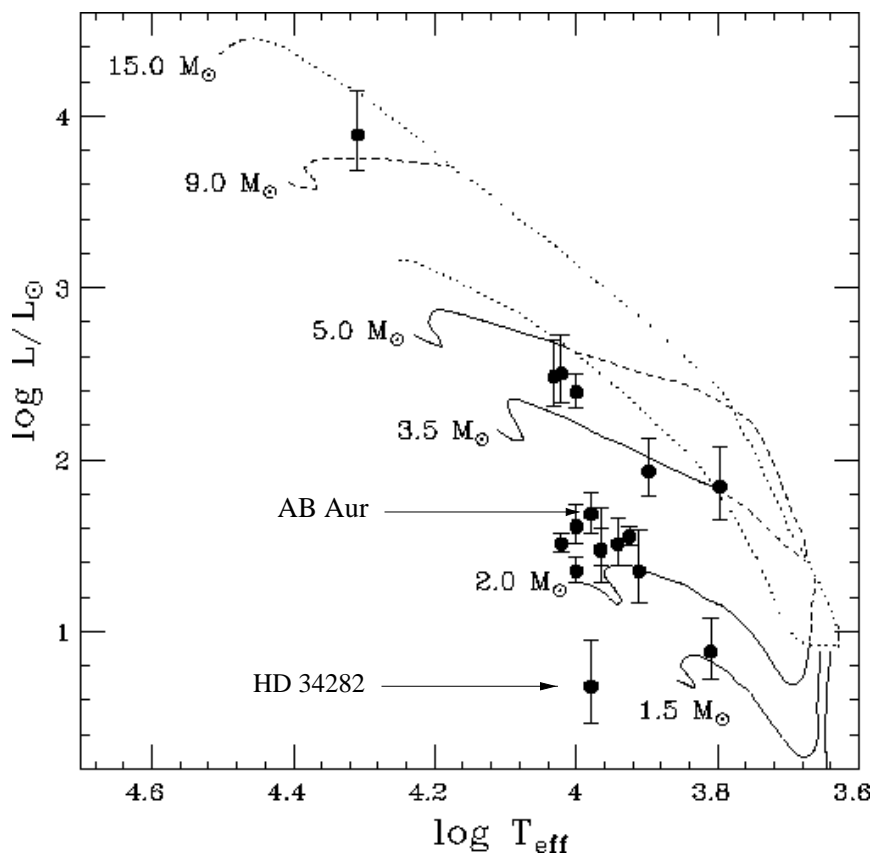

Fig. 3. Location of HD 34282 in a HR diagram for a distance $D=$ 160 pc (Fig. 1 of van den Ancker et al. 1998).

depletion factor $f\left({ }^{12} \mathrm{CO}\right)$ with respect to $X_{\mathrm{TMC} 1}^{12}: f\left({ }^{12} \mathrm{CO}\right) \leq$ $n_{100}($ dust $) / n_{100}(\mathrm{CO}) \sim 70$.

\section{Discussion}

\subsection{Stellar mass}

We have checked that the rotation pattern is almost Keplerian, with an inferred value of the rotation exponent $v=0.48 \pm 0.03$ as shown in Fig. 1d. (We will then assume $v=0.5$ in the final modelling.)

Simon et al. (2000) have shown that, in such a case, the dynamical mass (derived from $\mathrm{CO}$ modeling) provides an accurate measurement of the mass of the central star which scales as the distance $D$. Assuming a distance $D=160 \mathrm{pc}$, we derive a star mass $M_{160}=0.87 \pm 0.17 M_{\odot}$.

\subsection{Where is HD 34282 ?}

This low mass value is unexpected for an A0 type star. In addition, the luminosity derived from the photometric measurements of van den Ancker et al. (1998) is $L_{160}=4.7 L_{\odot}$, for a

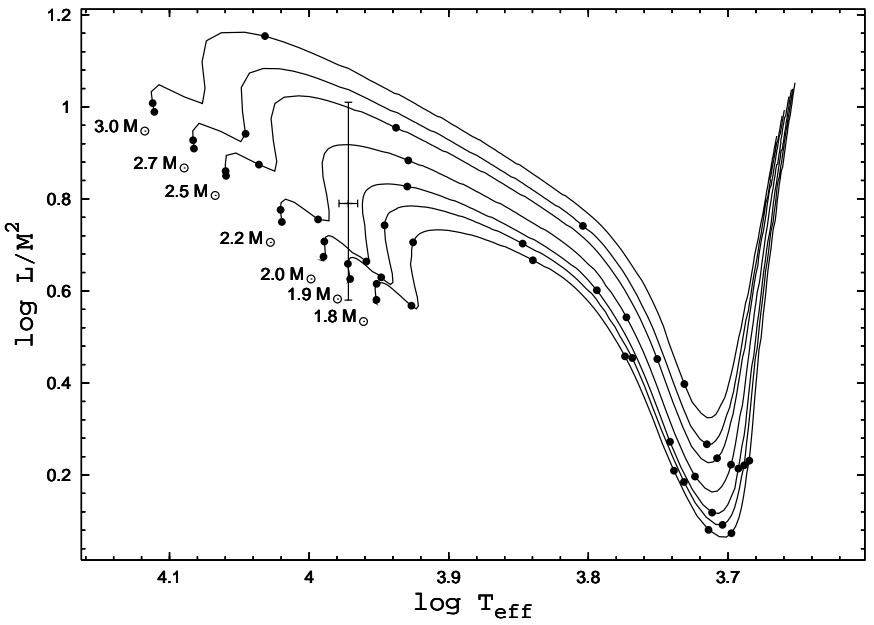

Fig. 4. Distance-independent diagram. Evolution tracks are from Siess et al. (2000) (available at http://www-laog.obs.ujfgrenoble.fr/activites/starevol/evol.html); dots are separated by 1 million years. Spectral-type A0.5 was converted to photospheric effective temperature using the results of Kenyon \& Hartmann (1995) and an error bar of half a subclass was added in each direction.

distance of $160 \mathrm{pc}$. The implied location of HD 34282 in the $\mathrm{H}-\mathrm{R}$ diagram places it at a totally different position to that of similar stars, such as AB Aur, and is incompatible with stellar evolution tracks (see Fig. 3). The spectral type determination by Cannon \& Mayall (1949) in the Henry Draper Catalog had been questioned by van den Ancker et al. (1998), but was subsequently confirmed by Gray \& Corbally (1998), who found the HD 34282 spectral type to be A0.5. Both "anomalies" point toward a revision of the star distance.

As the luminosity, $L$, scales as $D^{2}$, and the dynamical mass $M$ as $D$, plotting HD 34282 in a "distance-independent" evolution diagram $\left(L / M^{2}\right.$ vs. spectral type $)$ allows a direct comparison with theoretical evolution tracks (see Fig. 4). The error bars on the luminosity derived by van den Ancker et al. (1998) $\left(\log L_{160}=0.68_{-0.21}^{+0.27}\right)$ are dominated by the distance uncertainty. Assuming an arbitrary $10 \%$ uncertainty on the luminosity, we derive the error bars for $L / M^{2}$ plotted in Fig. 4 .

In this diagram, the star location corresponds to a stellar mass $M_{*}=2.1_{-0.2}^{+0.4} M_{\odot}$, in better agreement with its A0 spectral type. From the dynamical mass $\mathrm{M}_{160}$, we derive that actual distance of the star is $D=160 \times\left(M_{*} / M_{160}\right)=400_{-100}^{+170} \mathrm{pc}$. The distance derived from Hipparcos measurements on parallax (6.1 \pm 1.6 mas, van den Ancker et al. 1998) is more than $2 \sigma$ from our distance determination. From reddening considerations, Sylvester et al. (1996) derived a distance of $547 \mathrm{pc}$, somewhat higher but still consistent with our determination. It should be noticed that considering its position, its distance, and its systemic velocity, HD 34282 is probably associated with Orion A (Green \& Padman 1993).

\subsection{Distance revisited model}

At a distance $D=400 \mathrm{pc}$, the $\chi^{2}$ analysis leads to the best parameters given in Table 2 . 
Table 2. Best parameters for the HD 34282 disk (CO $J=2 \rightarrow 1$ data and continuum).

\begin{tabular}{|c|c|c|c|}
\hline Assumed Distance & $D(\mathrm{pc})=$ & 400 & \\
\hline Systemic velocity & $V_{\mathrm{LSR}}\left(\mathrm{km} \mathrm{s}^{-1}\right)=$ & -2.35 & \pm 0.02 \\
\hline Orientation & $\mathrm{PA}=$ & 25 & $\pm 2^{\circ}$ \\
\hline Inclination & $i=$ & 56 & $\pm 3^{\circ}$ \\
\hline Outer radius & $R_{\text {out }}(\mathrm{AU})=$ & 835 & \pm 20 \\
\hline Turbulent width & $\Delta v\left(\mathrm{~km} \cdot \mathrm{s}^{-1}\right)=$ & 0.11 & \pm 0.03 \\
\hline \multicolumn{4}{|c|}{ Abundance \& $\mathrm{H}_{2}$ Density law: $n(r)=n_{100}\left(\frac{r}{100 \mathrm{AU}}\right)^{-s}$} \\
\hline${ }^{12} \mathrm{CO}$ reference ${ }^{a}$ & $X_{\mathrm{TMC} 1}^{12}=$ & $7 \times 10^{-5}$ & - \\
\hline${ }^{12} \mathrm{CO}$ abundance & $X\left({ }^{12} \mathrm{CO}\right)>$ & $1 \times 10^{-6}$ & - \\
\hline${ }^{12} \mathrm{CO}$ depletion & $f\left({ }^{12} \mathrm{CO}\right)<$ & 70 & - \\
\hline & Density & & \\
\hline at $100 \mathrm{AU}$ & $n_{100}\left(\mathrm{~cm}^{-3}\right)=$ & $1.8 \times 10^{9}$ & \pm 0.6 \\
\hline exponent & $s=$ & 2.45 & \pm 0.35 \\
\hline \multicolumn{4}{|c|}{ Temperature law: $\quad T(r)=T_{100}\left(\frac{r}{100 \mathrm{AU}}\right)^{-q}$} \\
\hline \multirow{3}{*}{$\begin{array}{c}\text { Temperature } \\
\text { at } 100 \mathrm{AU} \\
\text { exponent }\end{array}$} & & & \\
\hline & $T_{100}(\mathrm{~K})=$ & 46 & \pm 5 \\
\hline & $q=$ & 0.52 & \pm 0.08 \\
\hline \multicolumn{4}{|c|}{ Velocity law: $\quad V(r)=V_{100}\left(\frac{r}{100 \mathrm{AU}}\right)^{-v}$} \\
\hline \multirow{3}{*}{$\begin{array}{l}\text { Velocity } \\
\text { at } 100 \mathrm{AU} \\
\text { exponent }\end{array}$} & & & \\
\hline & $V_{100}\left(\mathrm{~km} \mathrm{~s}^{-1}\right)=$ & 4.6 & \pm 0.2 \\
\hline & $v=$ & 0.48 & \pm 0.03 \\
\hline Stellar mass & $\mathrm{M}_{*}\left(M_{\odot}\right)=$ & 2.35 & \pm 0.2 \\
\hline \multicolumn{4}{|c|}{ Surface Density law: $\Sigma(r)=\Sigma_{100}\left(\frac{r}{100 \mathrm{AU}}\right)^{-p}$} \\
\hline \multirow{3}{*}{$\begin{array}{l}\text { Surface Density } \\
\text { at } 100 \mathrm{AU}\end{array}$} & & & \\
\hline & $\Sigma_{100}\left(\mathrm{~cm}^{-2}\right)=$ & $6 \times 10^{23}$ & \pm 2.2 \\
\hline & $\Sigma_{100}\left(\mathrm{~g} \mathrm{~cm}^{-2}\right)=$ & 2.3 & \pm 0.8 \\
\hline exponent & $p=$ & 1.2 & \pm 0.3 \\
\hline \multicolumn{4}{|c|}{ Scale Height law: $\quad H(r)=H_{100}\left(\frac{r}{100 \mathrm{AU}}\right)^{+h}$} \\
\hline \multicolumn{4}{|l|}{ Scale Height } \\
\hline at $100 \mathrm{AU}$ & $H_{100}(\mathrm{AU})=$ & 13 & - \\
\hline exponent & $h=$ & 1.24 & - \\
\hline \multicolumn{4}{|c|}{ Dust: $\kappa_{v}=\kappa_{o} \times\left(\frac{v}{10^{12} \mathrm{~Hz}}\right)^{\beta}$} \\
\hline Absorption law & $\kappa_{o}=$ & 0.1 & - \\
\hline Dust exponent & $\beta=$ & 1.15 & \pm 0.20 \\
\hline Dust disk size & $R_{\mathrm{d}}(\mathrm{AU})>$ & 700 & - \\
\hline total mass & $M_{\mathrm{d}}\left(M_{\odot}\right)=$ & $0.11_{-0.05}^{+0.09}$ & \\
\hline
\end{tabular}

The errors are the $1 \sigma$ formal errors from the $\chi^{2}$ fit, and do not take into account the distance uncertainty.

${ }^{a} X_{\mathrm{TMCl}}$, the ${ }^{12} \mathrm{CO}$ abundance in TMC 1 is taken from Cernicharo \& Guélin (1987).

The disk outer radius $R_{\text {out }}$ is $835 \mathrm{AU}$, among the largest known disks (e.g. DM Tau, GG Tau).

It should be noticed that both the disk inclination angle of $i=56^{\circ} \pm 3^{\circ}\left(0^{\circ}\right.$ means pole-on $)$ and the measured photometric variability $(\Delta V \simeq 2.5)$ of HD 34282 are compatible with the UX Ori model from Natta \& Whitney (2000).

At $100 \mathrm{AU}$, the disk has $T_{100}=46 \mathrm{~K}$ which is hotter than found for T Tauri stars such as DM Tau or GM Aur (Dutrey et al. 1998), which have similar radial variations of the temperature $(q=0.52 \pm 0.08)$. Assuming the revised luminosity of
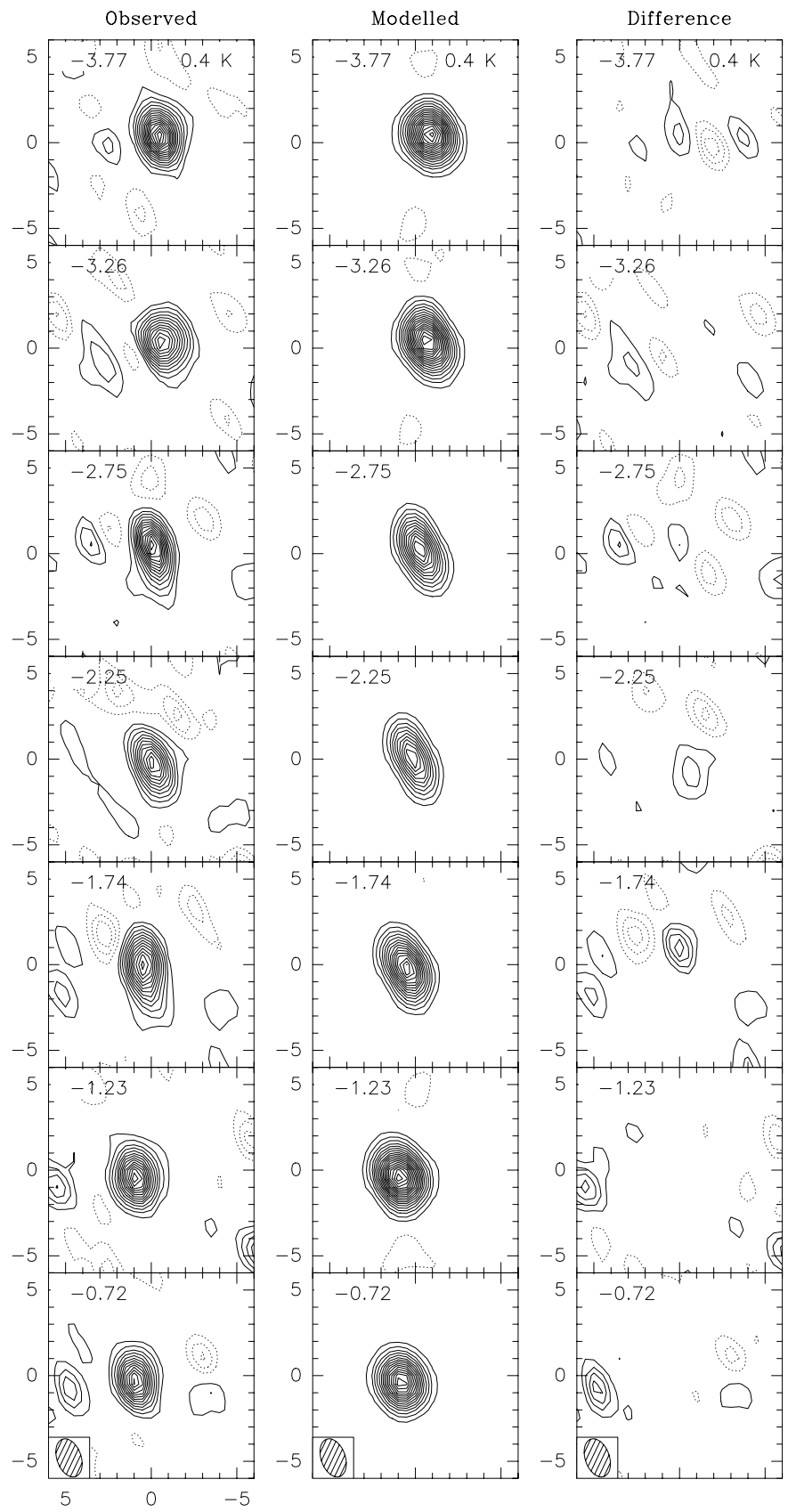

Fig. 5. Left: ${ }^{12} \mathrm{CO} \quad J=2 \rightarrow 1$ observations. Middle: best model. Right: difference. Contours represents $50 \mathrm{mJy} / \mathrm{beam}$, corresponding to a temperature of $0.35 \mathrm{~K}$, or $1.5 \sigma$. The channel velocity from top to bottom is: $-3.77,-3.26,-2.75,-2.25 .-1.74,-1.23-0.72 \mathrm{~km} \mathrm{~s}^{-1}$.

$29 L_{\odot}$, the blackbody temperature at $100 \mathrm{AU}$ would be around $50 \mathrm{~K}$. The temperature law of the disk is therefore compatible with a flared disk heated by the central star, e.g. (Chiang \& Goldreich 1997).

Assuming $T_{100}=46 \mathrm{~K}$ and $q=0.52$, we derive the surface density $\Sigma_{100} \simeq 6.5 \times 10^{23} \mathrm{~cm}^{-2}$ and $p \simeq 1.2$ from the dust analysis (see Fig. 2). With $p \simeq 1.2$, the surface density law is relatively shallow, as found in the previous continuum analysis of T Tauri disks by Dutrey et al. (1996).

The disk mass derived from modeling of the continuum emission is $M_{\mathrm{d}}=0.11_{-0.05}^{+0.09} M_{\odot}$ (for a gas-to-dust ratio of 
100). Such a massive disk is in agreement with previous results which suggest that there is a tendency for intermediate-mass stars to have more massive disks (Natta et al. 2000).

\section{Summary}

The disk surrounding the A0 PMS star HD 34282 has been mapped by the IRAM array in ${ }^{12} \mathrm{CO} J=2 \rightarrow 1$ and in continuum at 3.4 and $1.3 \mathrm{~mm}$. Standard disk modeling of the molecular emission, combined with dust thermal emission analysis and stellar distance-independent evolution tracks, allow us to conclude that:

- The CO disk, resolved by the interferometric observations, is in Keplerian rotation.

- The disk outer radius is large, with $R_{\text {out }}=835 \pm 20 \mathrm{AU}$.

- Its temperature law, derived from ${ }^{12} \mathrm{CO} J=2 \rightarrow 1$ line is in agreement with a flared disk heated by the central star.

- The disk continuum emission is optically thin and has a spectral index $\beta \simeq 1.1$.

- With an inferred total mass of $0.11_{-0.05}^{+0.09} M_{\odot}$, the disk is relatively massive.

- The stellar mass is $2.1_{-0.2}^{+0.4} M_{\odot}$ (as expected for a A0.5 spectral type star) and the star distance is $D=400_{-100}^{+170} \mathrm{pc}$, at a little bit more than $2 \sigma$ from the Hipparcos measurements. The revised luminosity would rather be around $L=$ $29_{-13}^{+30} L_{\odot}$.

In conclusion, the HD 34282 disk does not appear significantly different from the TTauri disks, except that it is more massive and somewhat hotter, as expected for an intermediate mass-star.

Acknowledgements. We warmly thank Dr. Mike Jura, who initiated this work during a visit of Anne Dutrey at UCLA, for his careful reading of the manuscript. Dimitri Pourbaix is acknowledged for providing us with many useful comments about Hipparcos analysis. Stéphane Guilloteau and Frédéric Gueth are thanked for fruitful discussions.
This research has made use of the SIMBAD database, operated at CDS, Strasbourg, France. We also would like to acknowledge the IRAM Plateau de Bure staff for providing the observations which were performed in service observing.

\section{References}

Beckwith, S. V. W., Sargent, A. I., Chini, R. S., \& Guesten, R. 1990, AJ, 99, 924

Bouwman, J., de Koter, A., van den Ancker, M. E., \& Waters, L. B. F. M. 2000, A\&A, 360, 213

Cannon, A. J., \& Mayall, M. W. 1949, Annals of Harvard College Observatory, 112, 1

Cernicharo, J., \& Guélin, M. 1987, A\&A, 176, 299

Chiang, E. I., \& Goldreich, P. 1997, ApJ, 490, 368+

Dutrey, A., Guilloteau, S., Duvert, G., et al. 1996, A\&A, 309, 493

Dutrey, A., Guilloteau, S., Prato, L., et al. 1998, A\&A, 338, L63

Dutrey, A., Guilloteau, S., \& Simon, M. 1994, A\&A, 286, 149

Grady, C. A., Woodgate, B., Bruhweiler, F. C., et al. 1999, ApJ, 523, L151

Gray, R. O., \& Corbally, C. J. 1998, AJ, 116, 2530

Greaves, J. S., Mannings, V., \& Holland, W. S. 2000, Icarus, 143, 155

Green, D. A., \& Padman, R. 1993, MNRAS, 263, 535

Guilloteau, S., \& Dutrey, A. 1998, A\&A, 339, 467

Kenyon, S. J., \& Hartmann, L. 1995, ApJS, 101, 117+

Koerner, D. W., Sargent, A. I., \& Beckwith, S. V. W. 1993, Icarus, 106,2

Malfait, K., Bogaert, E., \& Waelkens, C. 1998, A\&A, 331, 211

Mannings, V., Koerner, D. W., \& Sargent, A. I. 1997, Nature, 388, 555

Mannings, V., \& Sargent, A. I. 2000, ApJ, 529, 391

Natta, A., Grinin, V., \& Mannings, V. 2000, Protostars and Planets IV, 559

Natta, A., \& Whitney, B. A. 2000, A\&A, 364, 633

Siess, L., Dufour, E., \& Forestini, M. 2000, A\&A, 358, 593

Simon, M., Dutrey, A., \& Guilloteau, S. 2000, ApJ, 545, 1034

Sylvester, R. J., Skinner, C. J., Barlow, M. J., \& Mannings, V. 1996, MNRAS, 279, 915

van den Ancker, M. E., de Winter, D., \& Tjin A Djie, H. R. E. 1998, A\&A, 330, 145 